\title{
Targeting ALK2: An Open Science Approach to Developing Therapeutics for the Treatment of Diffuse Intrinsic Pontine Glioma
}

Deeba Ensan ${ }^{\dagger, \S}$ David Smil, ${ }^{\S}$ Carlos A. Zepeda-Velázquez, ${ }^{\S}$ Dimitrios Panagopoulos, $, \$, \ni, \varangle$ Jong Fu Wong, ${ }^{\ddagger}$ Eleanor P. Williams, ${ }^{\ddagger}$ Roslin Adamson, ${ }^{\ddagger}$ Alex N. Bullock, ${ }^{\star}$ Taira Kiyota, ${ }^{\S}$ Ahmed Aman ${ }^{\S, \zeta}$ Owen G. Roberts, ${ }^{, \mathrm{t}}$ Aled M. Edwards, ${ }^{\mathrm{tt}, \Im}$ Jeff A. O'Meara, ${ }^{\S, \mathrm{*}}$ Methvin B. Isaac, ${ }^{\S}$ Rima Al-awar ${ }^{*, \$}, \dagger$

$\dagger$ Department of Pharmacology and Toxicology, University of Toronto, Medical Sciences Building, Room 4207, 1 King's College Circle, Toronto, Ontario M5S 1A8, Canada ${ }^{\S}$ Drug Discovery Program, Ontario Institute for Cancer Research, 661 University Avenue, MaRS Centre, West Tower, Toronto, Ontario M5G 0A3, Canada

${ }^{\ni}$ Structural Genomics Consortium, University of Toronto, 101 College Street, MaRS Centre, South Tower, Toronto, Ontario M5G 1L7, Canada

${ }^{x}$ Department of Chemistry, Simon Fraser University, 8888 University Drive, Burnaby, British Columbia V5A 1S6, Canada

* Structural Genomics Consortium, University of Oxford, Old Road Campus, Roosevelt Drive, Oxford OX3 7DQ, United Kingdom

${ }_{\zeta}$ Leslie Dan Faculty of Pharmacy, University of Toronto, 144 College Street, Toronto, Ontario M5S 3M2, Canada

"t M4K Pharma Inc., 101 College Street, MaRS Centre, South Tower, Toronto, Ontario M5G 1L7, Canada

\section{- Supporting Information -}

1. NMR Spectra S2

2. Supplementary Tables S8 


\section{NMR Spectra}
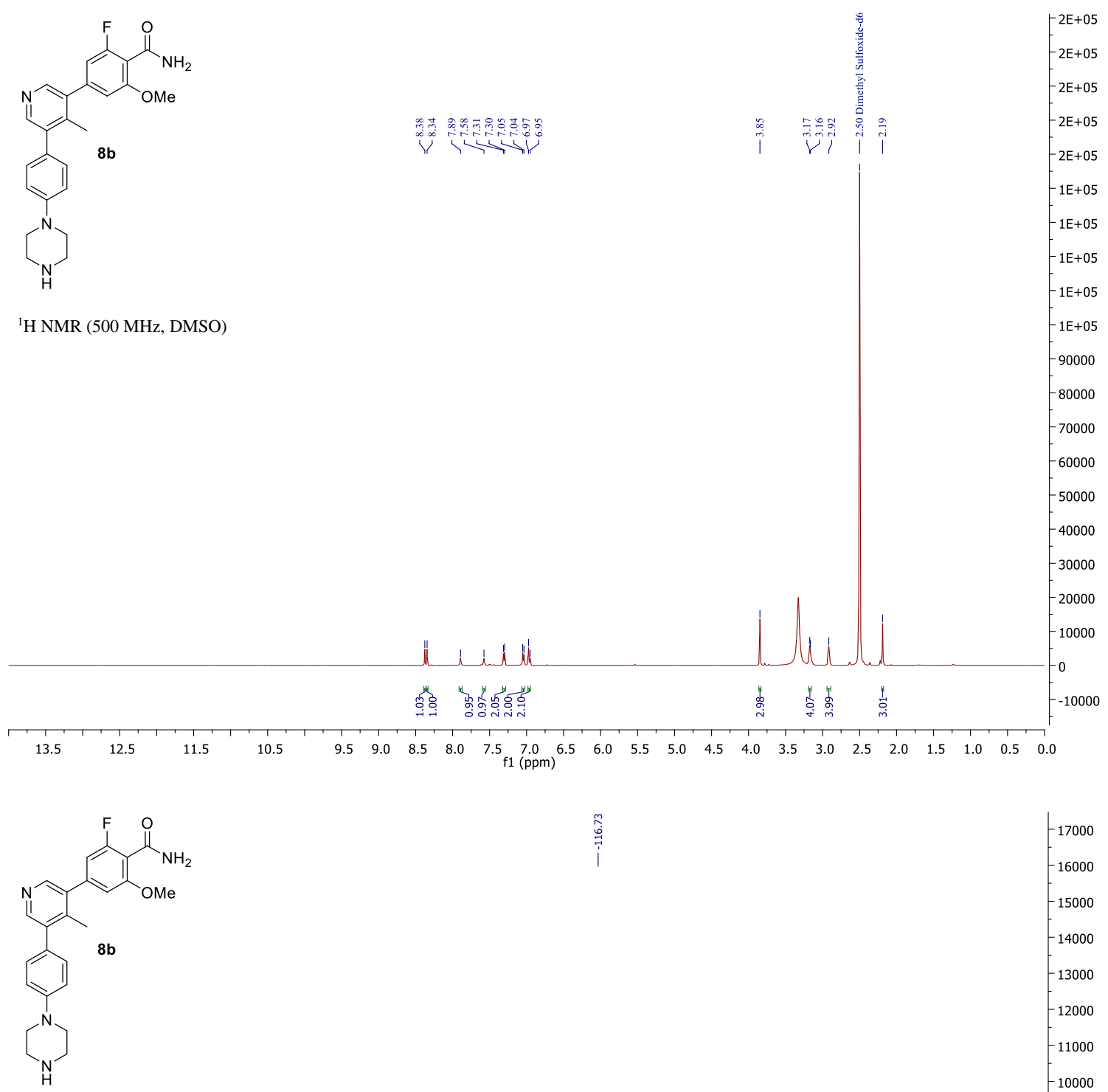

$\stackrel{n}{6}$
$\stackrel{0}{i}$
$i$

${ }^{19}$ F NMR (471 MHz, DMSO)

${ }^{1} \mathrm{H}$ NMR (500 MHz, DMSO)

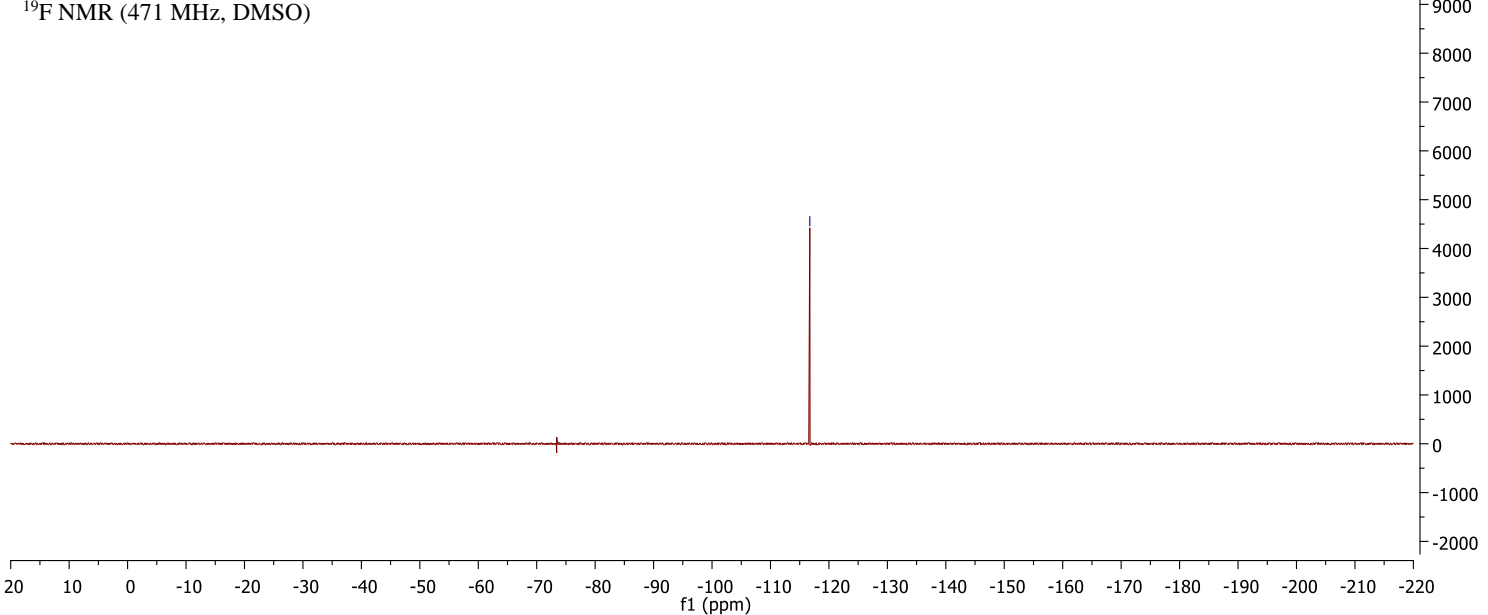



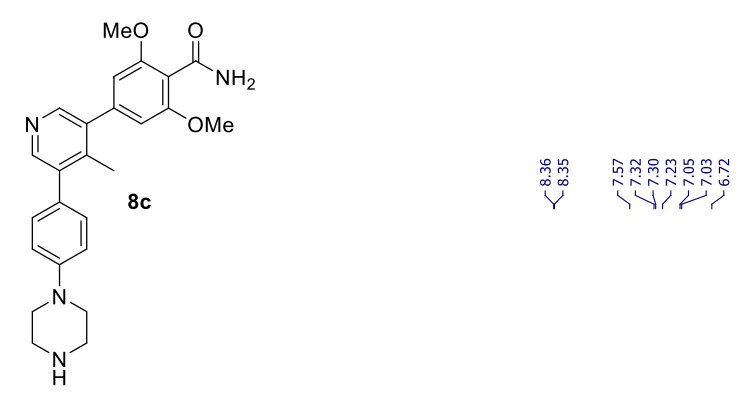

${ }^{1} \mathrm{H}$ NMR (500 MHz, DMSO)
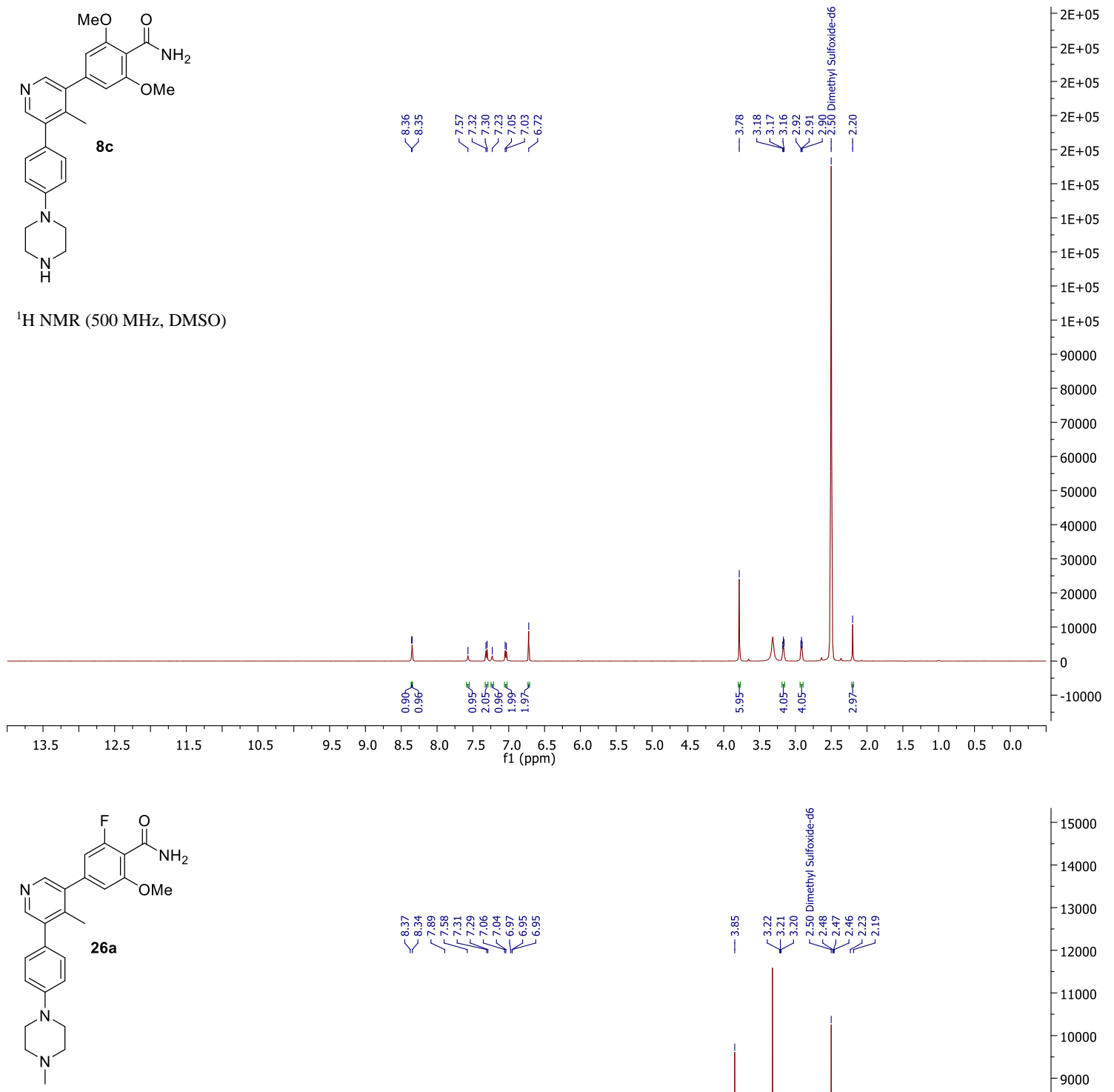

${ }^{1} \mathrm{H}$ NMR (500 MHz, DMSO)

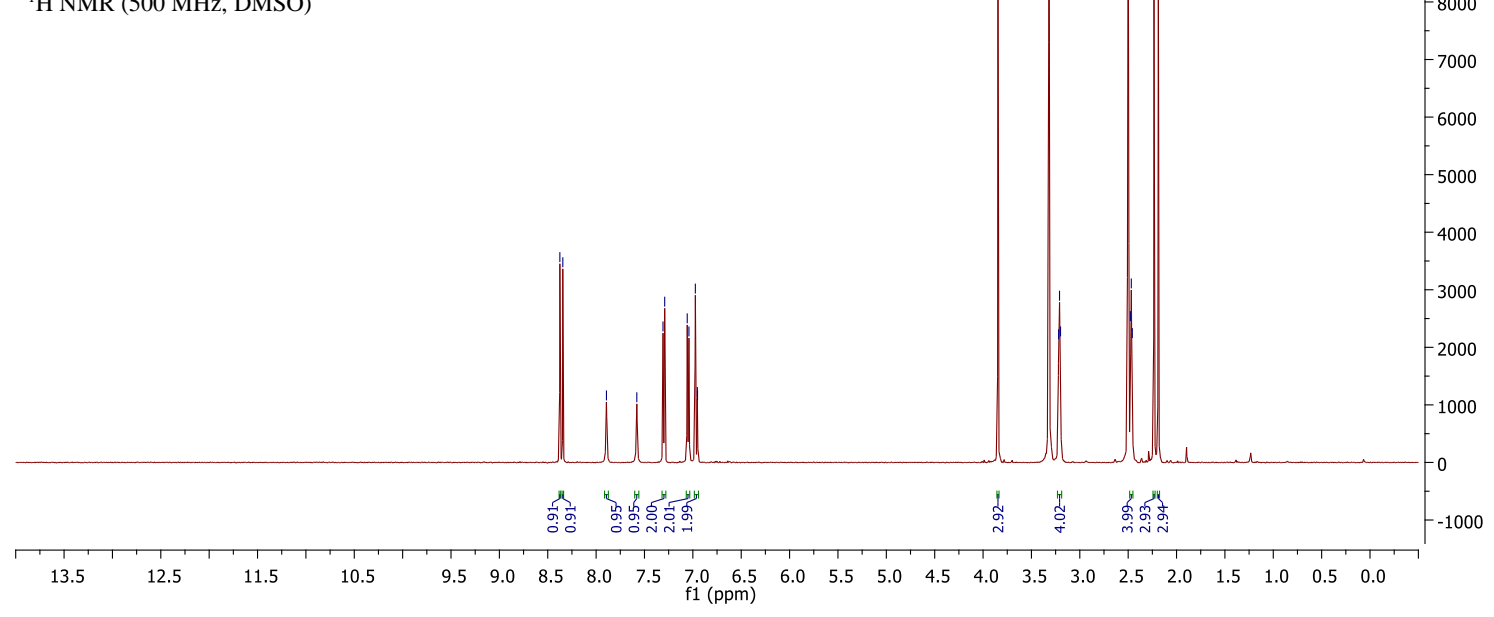




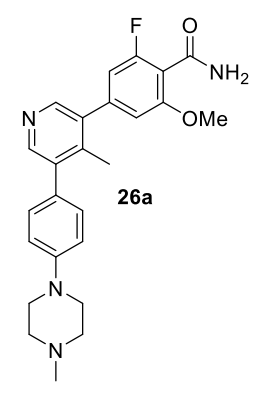

${ }^{19}$ F NMR (471 MHz, DMSO)
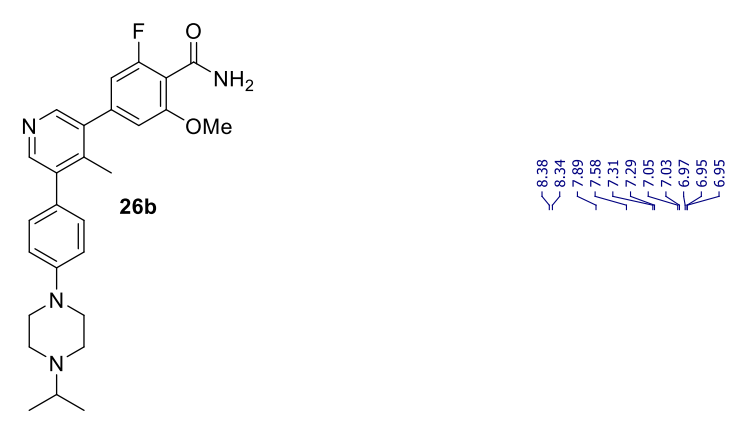

${ }^{1} \mathrm{H}$ NMR (500 MHz, DMSO)

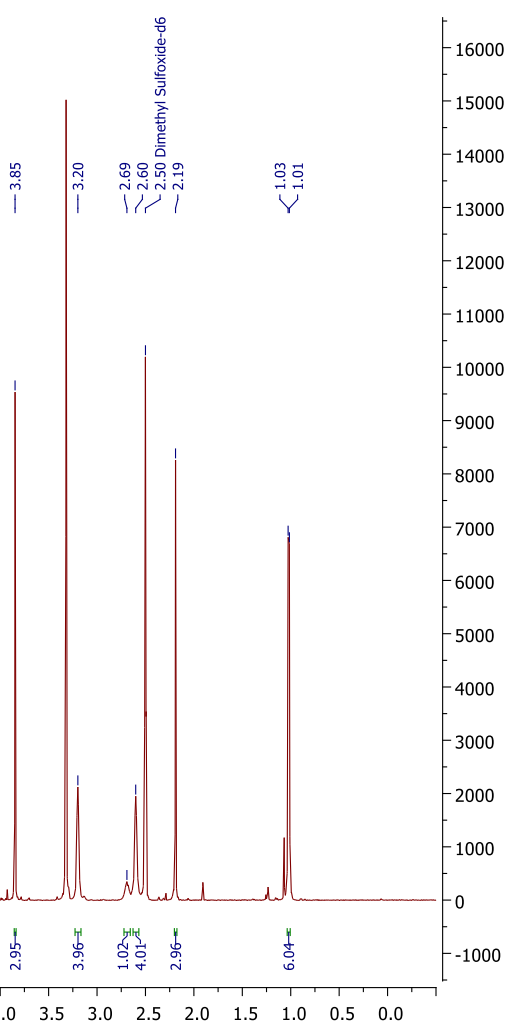



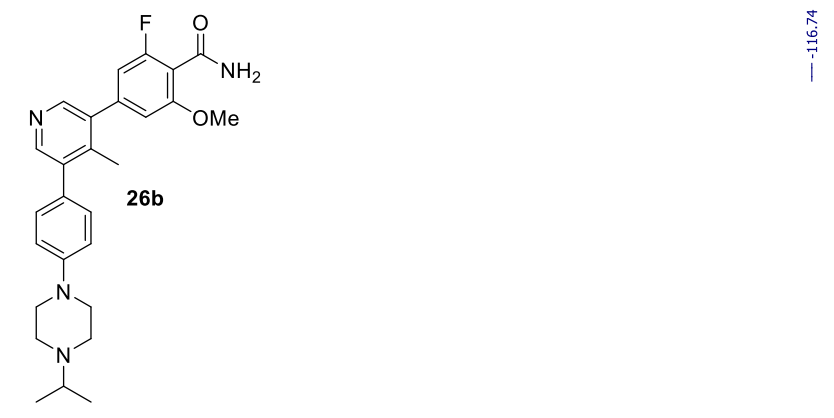

${ }^{19}$ F NMR (471 MHz, DMSO)
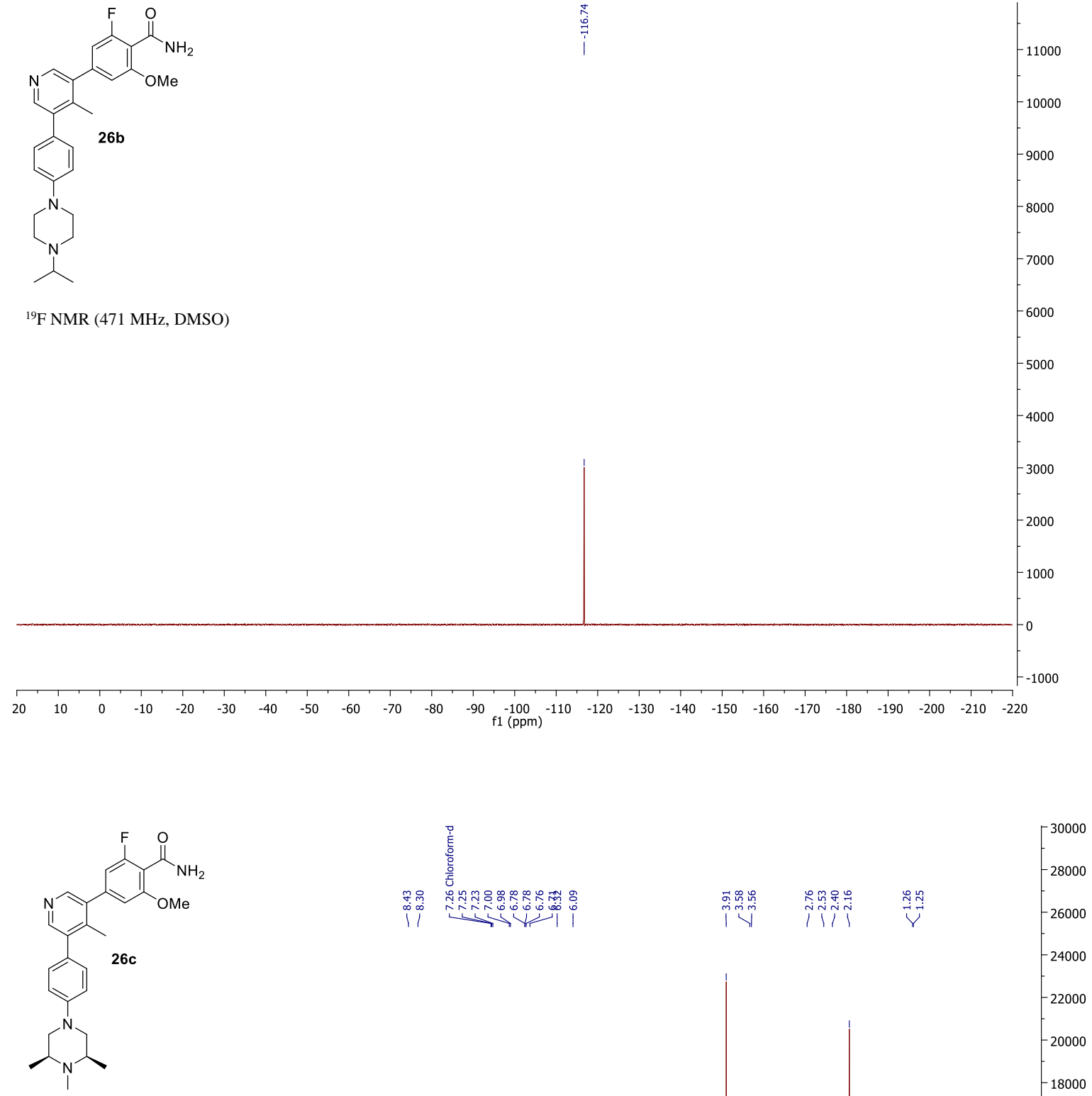

${ }^{1} \mathrm{H}$ NMR (500 MHz, $\mathrm{CDCl}_{3}$ )

24000

22000

$-20000$

$-18000$

16000

14000

12000

10000

$-8000$

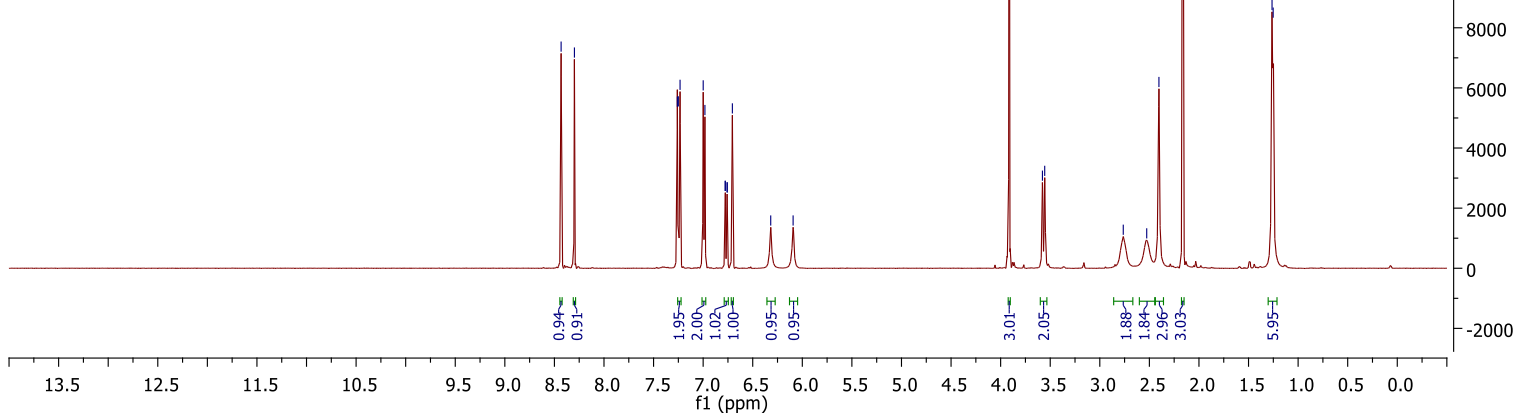



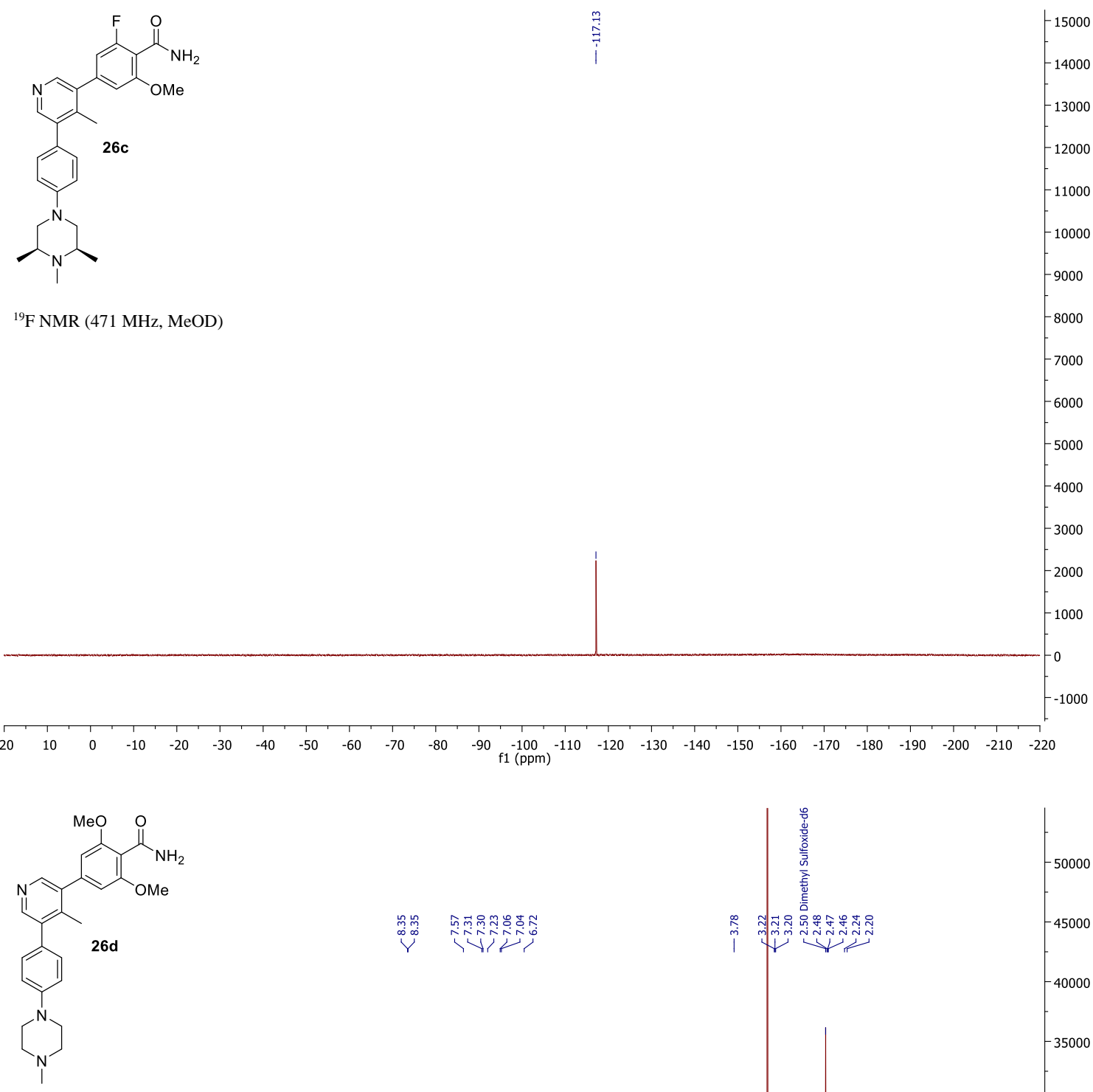

${ }^{1} \mathrm{H}$ NMR (500 MHz, DMSO)

${ }^{19} \mathrm{~F}$ NMR (471 MHz, MeOD) 

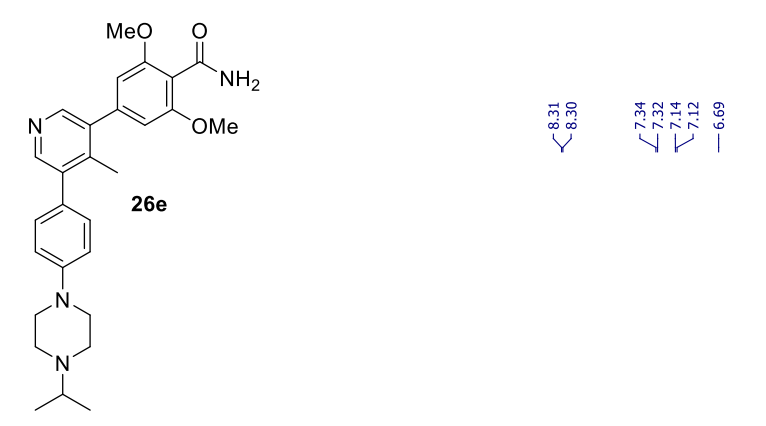

${ }^{1} \mathrm{H}$ NMR $(500 \mathrm{MHz}, \mathrm{MeOD})$
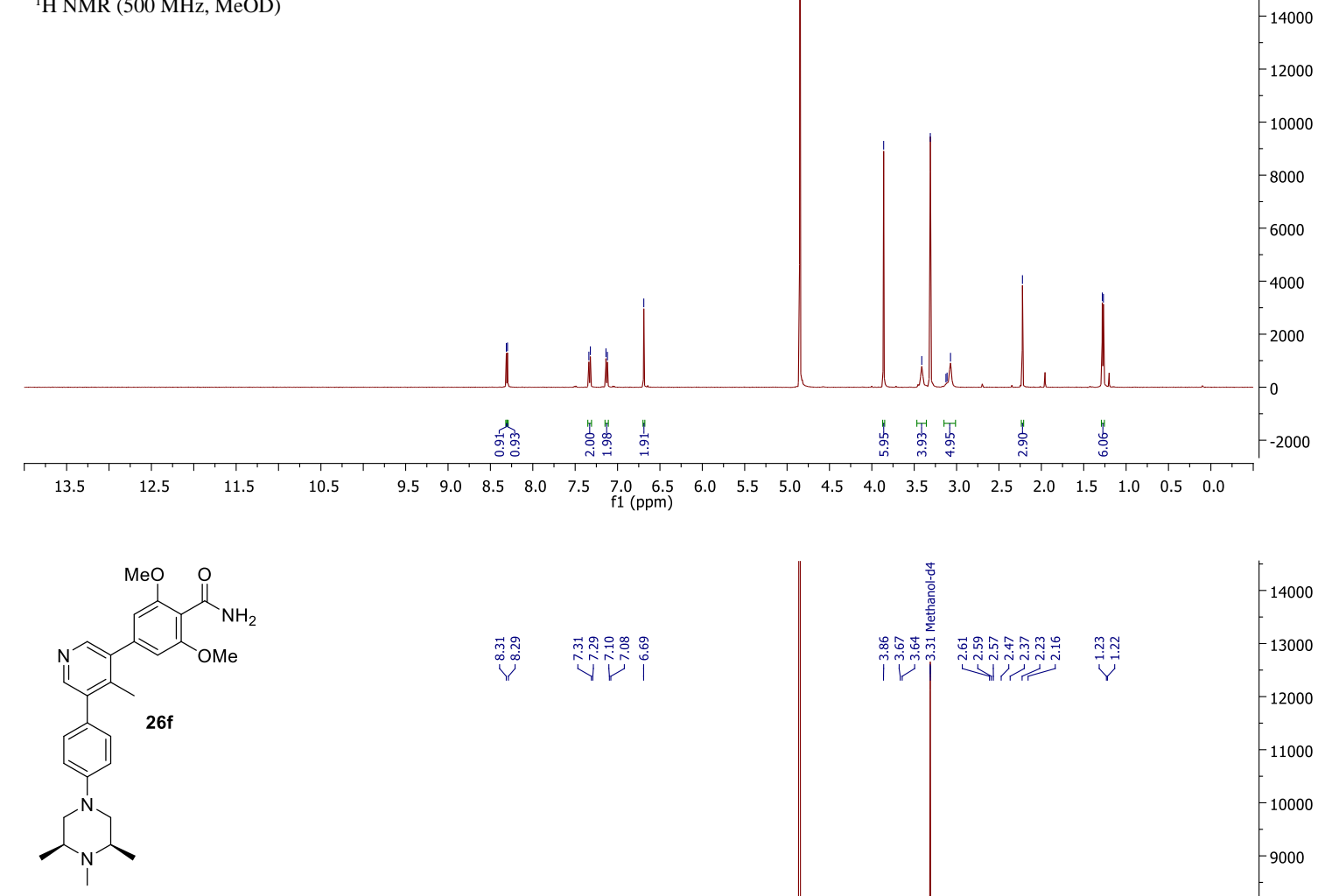

${ }^{1} \mathrm{H}$ NMR (500 MHz, MeOD)

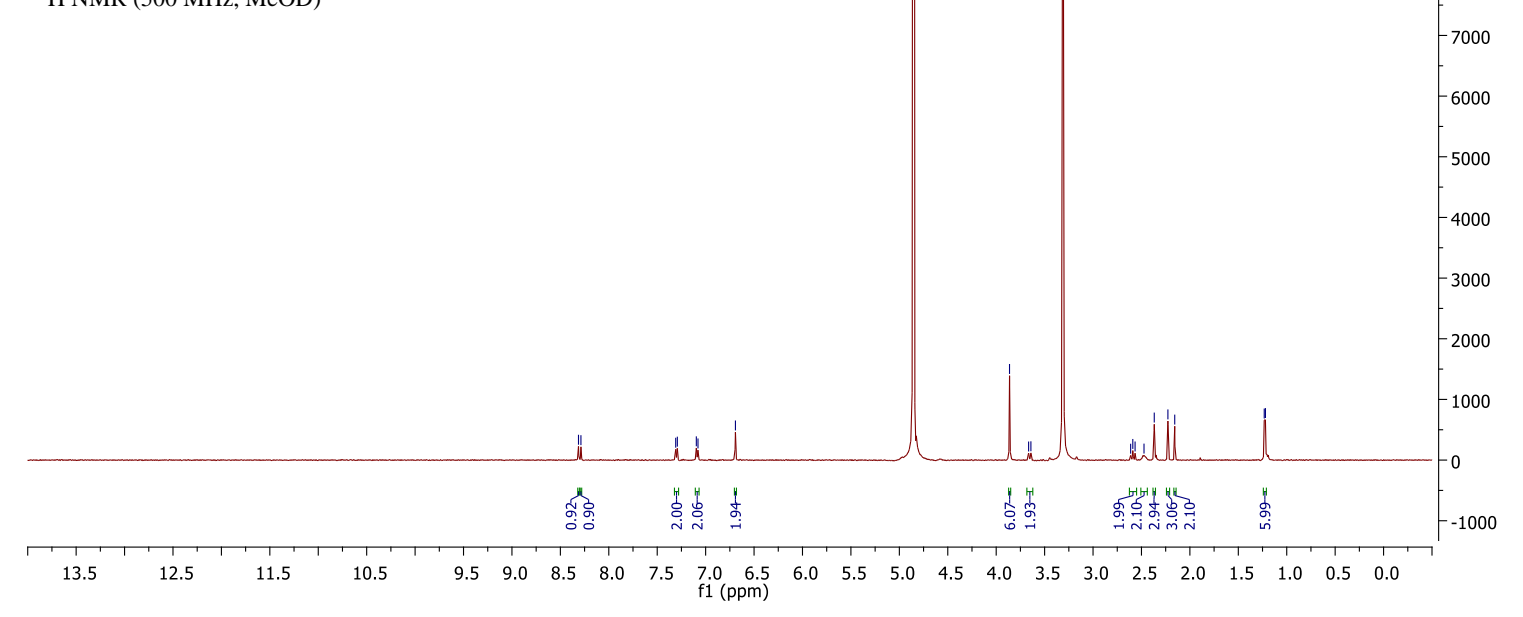




\section{Supplementary Tables}

Co-Crystallization of ALK2 with M4K2149: Phasing, Model Building, Refinement, and Validation

Table 1. Data collection and refinement statistics.

\begin{tabular}{|c|c|}
\hline & $\begin{array}{l}\text { ACVR1A with } \\
\text { M4K2149 }\end{array}$ \\
\hline 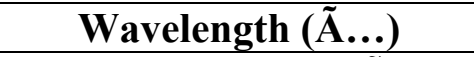 & 0.9763 \\
\hline Resolution range $(\tilde{\mathbf{A}} . .)$. & $\begin{array}{c}78.45-2.56(2.65- \\
2.56) \\
\end{array}$ \\
\hline Space group & $\mathrm{C} 121$ \\
\hline $\mathbf{a}, \mathbf{b}, \mathbf{c}(\AA)$ & $198.0,101.8,84.4$ \\
\hline$\alpha, \boldsymbol{\beta}, \gamma,\left(^{\circ}\right)$ & $90.0,111.4,90.0$ \\
\hline Total reflections & $671529(49767)$ \\
\hline Unique reflections & $50340(3699)$ \\
\hline Multiplicity & $13.3(13.5)$ \\
\hline Completeness $(\%)$ & $100.0(100.0)$ \\
\hline Mean I/sigma(I) & $8.8(1.3)$ \\
\hline Wilson B-factor $\left(\AA^{2}\right)$ & 58.84 \\
\hline R-merge & $0.197(3.197)$ \\
\hline $\mathrm{CC}(1 / 2)$ & $0.998(0.870)$ \\
\hline $\begin{array}{c}\text { Reflections used for } \\
\text { Refinement }\end{array}$ & 50137 \\
\hline R-work & 0.2242 \\
\hline R-free & 0.2717 \\
\hline $\begin{array}{c}\text { Number of non-hydrogen } \\
\text { atoms }\end{array}$ & 9146 \\
\hline macromolecules & 8941 \\
\hline ligands & 175 \\
\hline water & 30 \\
\hline Protein residues & 1152 \\
\hline RMS(bonds) (̊) & 0.002 \\
\hline RMS(angles) $\left(^{\circ}\right)$ & 0.47 \\
\hline $\begin{array}{c}\text { Ramachandran favored } \\
(\%)\end{array}$ & 96.21 \\
\hline $\begin{array}{c}\text { Ramachandran allowed } \\
(\%)\end{array}$ & 3.79 \\
\hline $\begin{array}{c}\text { Ramachandran outliers } \\
(\%)\end{array}$ & 0 \\
\hline
\end{tabular}




\begin{tabular}{|c|c|}
\hline Clashscore & 17.96 \\
\hline Average B-factor $\left(\AA^{\mathbf{2}}\right)$ & 73.66 \\
\hline macromolecules & 73.67 \\
\hline ligands & 75.18 \\
\hline solvent & 62.39 \\
\hline
\end{tabular}

Statistics for the highest-resolution shell are shown in parentheses

Table 2: Caco-2 permeability of 2,6-dimethoxybenzamide analogs

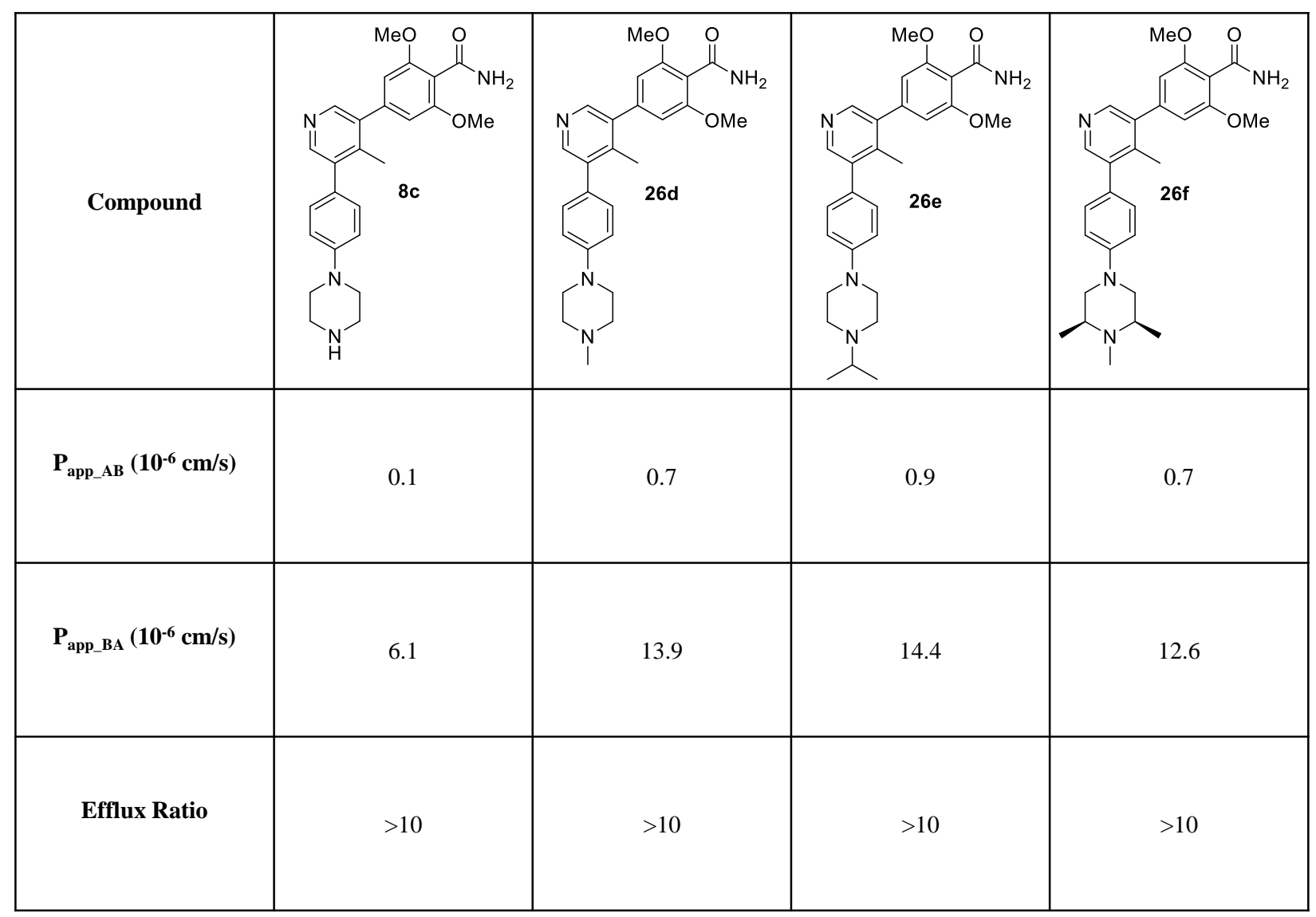

Table 3: HLM and MLM stability of lead amides

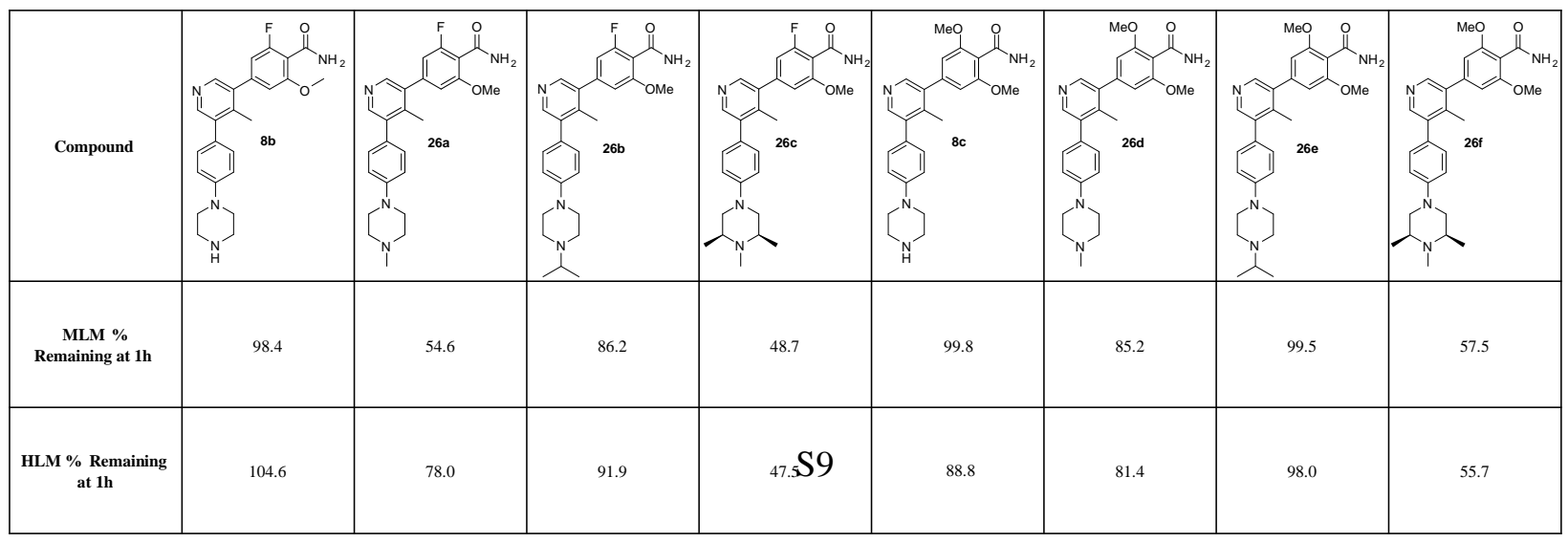


Table 4: Off-target activity of 2-fluoro-6-methoxybenzamide analogs (26a-c); CYP and hERG inhibition

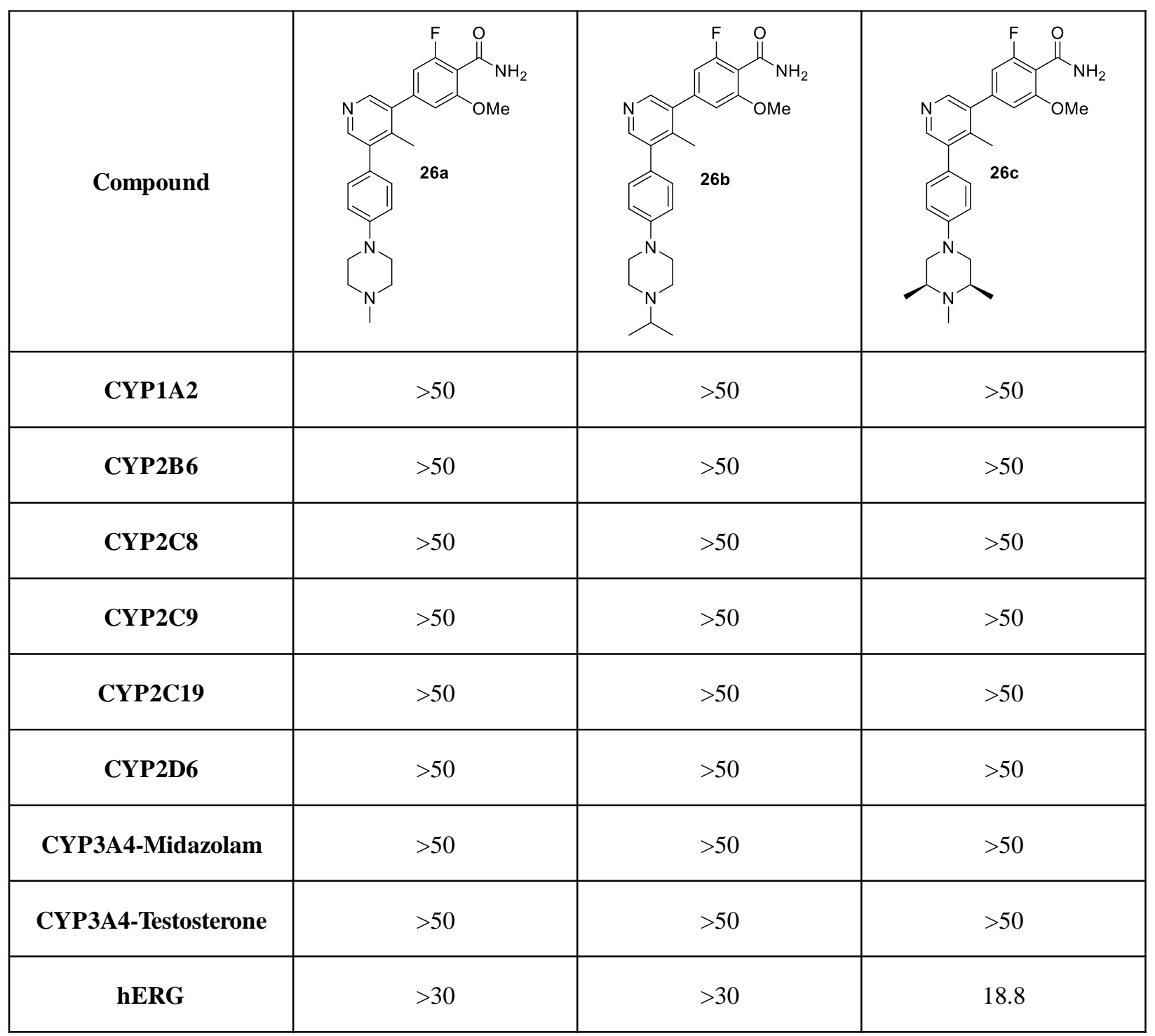

Table 5: 375-member kinase selectivity panel: \% enzyme activity at $1 \mu \mathrm{M}$

\begin{tabular}{|c|c|c|c|c|c|c|}
\hline \multirow{2}{*}{ Kinase } & \multicolumn{2}{|c|}{ 26b } & \multicolumn{2}{c|}{ 26a } & \multicolumn{2}{c|}{ 26c } \\
\cline { 2 - 6 } & Run 1 & Run 2 & Run 1 & Run 2 & Run 1 & Run 2 \\
\hline ABL1 & 84.48 & 80.78 & 75.19 & 75.12 & 83.92 & 82.07 \\
\hline ABL2/ARG & 98.76 & 98.48 & 97.35 & 95.77 & 97.10 & 96.59 \\
\hline ACK1 & 66.77 & 61.59 & 65.49 & 65.46 & 79.52 & 76.07 \\
\hline AKT1 & 101.90 & 99.01 & 105.74 & 102.39 & 105.40 & 104.50 \\
\hline AKT2 & 94.01 & 93.58 & 91.93 & 91.70 & 93.01 & 92.08 \\
\hline
\end{tabular}




\begin{tabular}{|c|c|c|c|c|c|c|}
\hline AKT3 & 98.54 & 97.15 & 94.96 & 94.73 & 98.68 & 98.56 \\
\hline ALK & 103.35 & 102.24 & 99.92 & 99.31 & 100.77 & 96.51 \\
\hline ALK1/ACVRL1 & 2.90 & 2.55 & 2.93 & 2.65 & 4.67 & 4.38 \\
\hline ALK2/ACVR1 & 7.69 & 5.67 & 9.92 & 8.63 & 13.70 & 11.70 \\
\hline ALK3/BMPR1A & 39.92 & 39.34 & 40.21 & 38.35 & 50.46 & 49.95 \\
\hline ALK4/ACVR1B & 81.34 & 79.81 & 68.83 & 68.79 & 82.37 & 81.71 \\
\hline ALK5/TGFBR1 & 84.09 & 83.42 & 82.46 & 82.28 & 97.24 & 96.60 \\
\hline ALK6/BMPR1B & 27.92 & 27.02 & 18.19 & 17.03 & 30.16 & 30.06 \\
\hline ARAF & 52.62 & 51.73 & 29.31 & 28.83 & 48.15 & 43.32 \\
\hline ARK5/NUAK1 & 92.50 & 92.31 & 97.59 & 90.26 & 96.00 & 93.75 \\
\hline ASK1/MAP3K5 & 101.03 & 99.17 & 95.53 & 94.20 & 97.79 & 96.09 \\
\hline Aurora A & 93.85 & 91.68 & 100.62 & 97.24 & 102.45 & 101.37 \\
\hline AURORA B & 112.26 & 110.21 & 102.08 & 100.58 & 105.03 & 104.62 \\
\hline Aurora C & 100.58 & 98.40 & 105.38 & 103.00 & 100.02 & 99.34 \\
\hline AXL & 81.11 & 81.04 & 81.85 & 81.59 & 91.27 & 86.95 \\
\hline BLK & 101.20 & 100.60 & 97.21 & 95.94 & 103.82 & 102.50 \\
\hline BMPR2 & 94.33 & 94.05 & 89.10 & 88.40 & 103.72 & 100.11 \\
\hline BMX/ETK & 102.78 & 102.35 & 103.19 & 102.04 & 101.61 & 100.72 \\
\hline BRAF & 44.45 & 44.03 & 28.84 & 28.77 & 41.09 & 40.52 \\
\hline BRK & 65.32 & 64.33 & 58.34 & 58.28 & 73.93 & 73.52 \\
\hline BRSK1 & 87.89 & 84.48 & 84.66 & 83.89 & 83.95 & 78.18 \\
\hline BRSK2 & 73.81 & 71.41 & 61.34 & 60.95 & 77.89 & 76.84 \\
\hline BTK & 97.71 & 97.62 & 95.31 & 94.35 & 96.55 & 94.07 \\
\hline c-Kit & 80.81 & 76.90 & 74.10 & 74.03 & 75.75 & 74.89 \\
\hline c-MER & 96.98 & 95.70 & 100.00 & 99.93 & 101.13 & 96.34 \\
\hline c-MET & 98.44 & 98.41 & 100.40 & 98.28 & 107.02 & 106.76 \\
\hline c-Src & 98.33 & 98.08 & 96.63 & 96.22 & 100.08 & 97.10 \\
\hline CAMK1a & 94.69 & 91.83 & 94.86 & 92.25 & 97.52 & 96.82 \\
\hline CAMK1b & 100.78 & 99.74 & 97.89 & 95.63 & 99.36 & 99.02 \\
\hline CAMK1d & 100.93 & 98.72 & 98.48 & 98.20 & 99.93 & 97.72 \\
\hline CAMK1g & 98.99 & 97.85 & 102.44 & 99.10 & 107.90 & 107.66 \\
\hline CAMK2a & 89.93 & 89.17 & 85.47 & 83.69 & 96.65 & 93.72 \\
\hline CAMK2b & 89.04 & 86.76 & 83.94 & 83.40 & 92.83 & 91.32 \\
\hline CAMK2d & 93.56 & 93.45 & 95.08 & 94.73 & 96.21 & 95.15 \\
\hline CAMK2g & 96.32 & 96.27 & 102.51 & 100.65 & 109.89 & 107.66 \\
\hline CAMK4 & 103.80 & 101.77 & 100.25 & 98.68 & 103.35 & 100.90 \\
\hline CAMKK1 & 92.70 & 88.83 & 94.66 & 92.05 & 94.05 & 93.52 \\
\hline CAMKK2 & 79.40 & 79.29 & 71.74 & 70.52 & 76.42 & 75.78 \\
\hline CDC7/DBF4 & 98.14 & 94.08 & 92.20 & 91.84 & 94.17 & 93.55 \\
\hline
\end{tabular}




\begin{tabular}{|c|c|c|c|c|c|c|}
\hline CDK1/cyclin A & 84.48 & 83.71 & 94.73 & 92.50 & 90.77 & 89.52 \\
\hline CDK1/cyclin B & 90.35 & 89.90 & 95.67 & 92.35 & 94.04 & 91.98 \\
\hline CDK1/cyclin E & 100.40 & 99.30 & 98.29 & 97.19 & 92.40 & 91.92 \\
\hline $\begin{array}{c}\text { CDK14/cyclin Y } \\
\text { (PFTK1) }\end{array}$ & 97.79 & 97.41 & 98.41 & 98.24 & 99.84 & 99.61 \\
\hline $\begin{array}{c}\text { CDK16/cyclin Y } \\
\text { (PCTAIRE) }\end{array}$ & 93.46 & 91.35 & 93.35 & 92.85 & 92.26 & 91.60 \\
\hline $\begin{array}{c}\text { CDK17/cyclin Y } \\
\text { (PCTK2) }\end{array}$ & 94.88 & 93.99 & 96.13 & 95.45 & 100.57 & 99.91 \\
\hline $\begin{array}{c}\text { CDK18/cyclin Y } \\
\text { (PCTK3) }\end{array}$ & 101.52 & 100.66 & 102.15 & 100.43 & 99.05 & 97.60 \\
\hline CDK19/cyclin C & 82.42 & 82.07 & 79.08 & 77.81 & 87.08 & 85.09 \\
\hline CDK2/cyclin A & 92.33 & 92.29 & 99.96 & 97.95 & 99.98 & 98.65 \\
\hline CDK2/Cyclin A1 & 111.93 & 111.68 & 99.92 & 99.67 & 98.22 & 97.14 \\
\hline CDK2/cyclin E & 106.40 & 104.69 & 104.56 & 104.52 & 102.22 & 102.10 \\
\hline CDK2/cyclin E2 & 104.97 & 104.46 & 108.37 & 104.96 & 95.00 & 93.14 \\
\hline CDK2/cyclin O & 92.35 & 92.30 & 95.09 & 93.78 & 92.72 & 88.53 \\
\hline CDK3/cyclin E & 85.83 & 85.35 & 82.66 & 81.63 & 78.70 & 78.38 \\
\hline CDK3/cyclin E2 & 85.07 & 84.43 & 89.55 & 88.05 & 86.16 & 83.94 \\
\hline CDK4/cyclin D1 & 100.09 & 99.89 & 99.36 & 98.46 & 100.47 & 100.29 \\
\hline CDK4/cyclin D3 & 93.74 & 93.67 & 100.73 & 96.96 & 96.05 & 95.54 \\
\hline CDK5/P25 & 93.58 & 93.23 & 94.60 & 91.77 & 94.85 & 94.09 \\
\hline CDK5/p35 & 95.63 & 95.08 & 96.22 & 95.26 & 97.61 & 96.90 \\
\hline CDK6/cyclin D1 & 101.19 & 100.82 & 100.38 & 97.80 & 95.90 & 92.80 \\
\hline CDK6/cyclin D3 & 89.55 & 88.85 & 86.29 & 86.25 & 103.38 & 102.19 \\
\hline CDK7/cyclin H & 103.42 & 101.47 & 97.01 & 93.27 & 96.12 & 95.50 \\
\hline CDK8/cyclin C & 90.11 & 89.57 & 84.60 & 83.64 & 93.06 & 92.05 \\
\hline CDK9/cyclin K & 105.53 & 104.69 & 97.53 & 96.95 & 105.74 & 102.30 \\
\hline CDK9/cyclin T1 & 93.52 & 92.35 & 90.84 & 90.82 & 97.07 & 96.85 \\
\hline CDK9/cyclin T2 & 102.05 & 102.02 & 100.00 & 99.00 & 101.72 & 100.35 \\
\hline CHK1 & 106.02 & 105.34 & 98.22 & 94.26 & 116.91 & 100.95 \\
\hline CHK2 & 95.42 & 95.41 & 99.69 & 99.41 & 102.33 & 101.19 \\
\hline CK1a1 & 99.28 & 99.26 & 96.07 & 91.31 & 100.33 & 99.44 \\
\hline CK1a1L & 103.89 & 102.82 & 98.38 & 97.55 & 99.41 & 99.15 \\
\hline CK1d & 100.50 & 99.03 & 98.06 & 94.27 & 108.24 & 108.05 \\
\hline CK1epsilon & 95.99 & 91.70 & 93.82 & 93.07 & 94.74 & 90.08 \\
\hline CK1g1 & 61.14 & 60.03 & 57.68 & 55.41 & 71.74 & 69.61 \\
\hline CK1g2 & 73.25 & 70.17 & 77.96 & 75.72 & 86.97 & 85.61 \\
\hline CK1G3 & 71.45 & 69.81 & 65.92 & 64.87 & 78.82 & 77.64 \\
\hline CK2a & 88.72 & 85.73 & 92.50 & 91.32 & 87.19 & 86.32 \\
\hline CK2a2 & 98.13 & 91.24 & 95.26 & 92.27 & 101.45 & 98.98 \\
\hline
\end{tabular}




\begin{tabular}{|c|c|c|c|c|c|c|}
\hline CLK1 & 93.61 & 92.82 & 97.84 & 94.42 & 88.03 & 86.91 \\
\hline CLK2 & 88.31 & 87.08 & 91.72 & 91.71 & 90.33 & 85.73 \\
\hline CLK3 & 92.75 & 91.99 & 103.06 & 99.59 & 88.26 & 86.89 \\
\hline CLK4 & 94.89 & 93.34 & 88.92 & 85.58 & 92.56 & 91.14 \\
\hline СОТ1/MАР3К8 & 94.82 & 93.40 & 90.60 & 88.67 & 93.89 & 92.86 \\
\hline CSK & 94.12 & 92.13 & 99.55 & 99.36 & 103.67 & 102.04 \\
\hline CTK/MATK & 95.25 & 94.38 & 95.23 & 94.85 & 99.24 & 98.62 \\
\hline DAPK1 & 98.50 & 98.30 & 95.66 & 94.56 & 98.78 & 96.25 \\
\hline DAPK2 & 104.25 & 103.70 & 103.30 & 101.48 & 100.40 & 95.98 \\
\hline DCAMKL1 & 84.74 & 83.59 & 81.84 & 81.46 & 89.11 & 85.85 \\
\hline DCAMKL2 & 70.71 & 69.99 & 64.44 & 62.07 & 84.54 & 82.24 \\
\hline DDR1 & 40.20 & 39.34 & 32.12 & 31.23 & 57.22 & 55.50 \\
\hline DDR2 & 92.60 & 91.94 & 100.02 & 96.68 & 92.70 & 92.27 \\
\hline DLK/MAP3K12 & 94.50 & 94.38 & 93.71 & 91.76 & 99.18 & 97.93 \\
\hline DMPK & 111.79 & 110.69 & 108.78 & 108.75 & 105.34 & 99.12 \\
\hline DMPK2 & 87.02 & 85.28 & 83.84 & 82.52 & 87.55 & 86.97 \\
\hline DRAK1/STK17A & 97.03 & 96.54 & 96.37 & 95.60 & 96.60 & 95.69 \\
\hline DYRK1/DYRK1A & 57.20 & 56.39 & 43.26 & 43.07 & 62.42 & 60.05 \\
\hline DYRK1B & 53.64 & 52.79 & 40.65 & 39.80 & 59.24 & 58.52 \\
\hline DYRK2 & 97.52 & 95.78 & 96.34 & 95.06 & 100.45 & 98.44 \\
\hline DYRK3 & 93.39 & 92.56 & 90.42 & 88.30 & 96.03 & 96.01 \\
\hline DYRK4 & 97.15 & 96.54 & 102.84 & 102.49 & 101.22 & 100.24 \\
\hline EGFR & 101.33 & 101.05 & 96.02 & 95.66 & 95.30 & 93.91 \\
\hline EPHA1 & 97.99 & 97.40 & 97.49 & 96.36 & 93.32 & 89.93 \\
\hline EPHA2 & 102.33 & 98.97 & 102.55 & 97.38 & 108.18 & 104.56 \\
\hline EPHA3 & 101.11 & 100.47 & 95.66 & 94.70 & 101.16 & 98.11 \\
\hline EPHA4 & 101.47 & 99.89 & 95.31 & 91.81 & 99.30 & 98.76 \\
\hline EPHA5 & 94.67 & 94.61 & 92.21 & 90.37 & 92.65 & 90.66 \\
\hline EPHА6 & 103.23 & 101.59 & 104.89 & 103.54 & 105.77 & 103.73 \\
\hline EPHA7 & 102.78 & 102.43 & 100.53 & 98.85 & 98.39 & 98.36 \\
\hline EPHA8 & 102.03 & 95.79 & 95.26 & 94.54 & 97.48 & 97.01 \\
\hline EPHB1 & 91.48 & 91.06 & 95.49 & 91.99 & 92.59 & 89.37 \\
\hline ЕРНB2 & 96.21 & 95.97 & 93.91 & 92.89 & 98.85 & 97.40 \\
\hline EPHB3 & 94.79 & 92.25 & 95.50 & 95.16 & 98.92 & 96.08 \\
\hline EPHB4 & 91.05 & 85.87 & 94.68 & 93.42 & 91.55 & 91.07 \\
\hline ERBB2/HER2 & 87.21 & 85.10 & 89.74 & 89.07 & 96.83 & 95.40 \\
\hline ERBB4/HER4 & 100.83 & 100.25 & 99.50 & 99.40 & 94.94 & 93.88 \\
\hline ERK1 & 98.69 & 97.39 & 97.34 & 94.73 & 95.26 & 94.56 \\
\hline ERK2/MAPK1 & 89.35 & 88.25 & 92.54 & 91.58 & 89.78 & 87.49 \\
\hline
\end{tabular}




\begin{tabular}{|c|c|c|c|c|c|c|}
\hline ERK5/MAPK7 & 99.60 & 98.66 & 96.51 & 92.77 & 100.55 & 98.01 \\
\hline ERK7/MAPK15 & 90.48 & 89.98 & 86.79 & 85.69 & 98.32 & 96.45 \\
\hline ERN1/IRE1 & 98.26 & 96.56 & 92.80 & 92.47 & 90.99 & 90.44 \\
\hline ERN2/IRE2 & 104.16 & 103.94 & 97.51 & 97.32 & 98.87 & 96.29 \\
\hline FAK/PTK2 & 96.05 & 87.69 & 94.56 & 93.97 & 97.12 & 95.42 \\
\hline FER & 83.86 & 83.48 & 89.18 & 86.16 & 86.01 & 84.91 \\
\hline FES/FPS & 97.02 & 96.09 & 99.93 & 99.20 & 98.72 & 98.66 \\
\hline FGFR1 & 101.69 & 101.63 & 97.43 & 96.52 & 101.12 & 99.91 \\
\hline FGFR2 & 97.84 & 97.43 & 95.51 & 95.46 & 91.89 & 90.89 \\
\hline FGFR3 & 104.85 & 104.10 & 101.07 & 100.98 & 102.79 & 102.08 \\
\hline FGFR4 & 95.18 & 94.51 & 98.18 & 97.61 & 99.61 & 98.99 \\
\hline FGR & 93.41 & 91.32 & 83.07 & 74.69 & 89.43 & 86.35 \\
\hline FLT1/VEGFR1 & 98.17 & 95.86 & 107.26 & 105.29 & 107.93 & 104.04 \\
\hline FLT3 & 90.68 & 85.34 & 77.06 & 76.15 & 84.31 & 81.48 \\
\hline FLT4/VEGFR3 & 96.83 & 96.06 & 98.40 & 97.81 & 97.06 & 96.61 \\
\hline FMS & 49.28 & 48.82 & 82.24 & 75.66 & 79.55 & 78.83 \\
\hline FRK/PTK5 & 100.39 & 99.17 & 100.82 & 95.48 & 98.20 & 95.52 \\
\hline FYN & 121.72 & 119.07 & 99.86 & 97.69 & 97.01 & 96.30 \\
\hline GCK/MAP4K2 & 68.25 & 67.48 & 61.77 & 59.95 & 75.44 & 74.91 \\
\hline GLK/MAP4K3 & 83.93 & 83.55 & 88.42 & 88.16 & 90.29 & 88.71 \\
\hline GRK1 & 95.38 & 93.08 & 94.01 & 93.15 & 95.27 & 94.01 \\
\hline GRK2 & 106.03 & 105.76 & 105.04 & 103.58 & 100.58 & 99.17 \\
\hline GRK3 & 97.93 & 96.85 & 94.39 & 94.07 & 96.12 & 95.20 \\
\hline GRK4 & 91.85 & 90.10 & 80.43 & 79.15 & 97.14 & 92.15 \\
\hline GRK5 & 99.91 & 97.81 & 103.50 & 99.06 & 93.13 & 91.62 \\
\hline GRK6 & 104.08 & 103.22 & 108.53 & 107.43 & 97.07 & 94.12 \\
\hline GRK7 & 100.81 & 100.06 & 95.12 & 93.81 & 91.79 & 91.35 \\
\hline GSK3a & 102.65 & 100.51 & 101.64 & 100.80 & 102.24 & 101.44 \\
\hline GSK3b & 99.45 & 96.28 & 88.53 & 84.98 & 88.84 & 88.83 \\
\hline Haspin & 84.75 & 83.53 & 83.33 & 82.77 & 84.79 & 82.31 \\
\hline HCK & 95.09 & 93.44 & 93.59 & 91.96 & 91.82 & 88.06 \\
\hline HGK/MAP4K4 & 29.70 & 29.61 & 21.96 & 20.97 & 30.63 & 28.35 \\
\hline HIPK1 & 100.16 & 99.62 & 106.43 & 102.36 & 99.91 & 96.64 \\
\hline HIPK2 & 101.68 & 96.91 & 99.85 & 97.88 & 103.25 & 101.85 \\
\hline HIPK3 & 87.90 & 85.10 & 97.83 & 97.73 & 90.44 & 89.08 \\
\hline HIPK4 & 104.07 & 103.79 & 103.63 & 102.90 & 100.58 & 97.53 \\
\hline HPK1/MAP4K1 & 56.39 & 55.64 & 58.71 & 57.53 & 74.71 & 74.31 \\
\hline IGF1R & 100.72 & 99.60 & 99.22 & 98.31 & 96.08 & 94.80 \\
\hline IKKa/CHUK & 85.62 & 84.25 & 83.26 & 82.74 & 87.42 & 85.96 \\
\hline
\end{tabular}




\begin{tabular}{|c|c|c|c|c|c|c|}
\hline IKKb/IKBKB & 108.96 & 108.24 & 104.06 & 101.39 & 101.07 & 100.59 \\
\hline IKKe/IKBKE & 92.20 & 82.11 & 91.06 & 87.51 & 96.73 & 94.75 \\
\hline IR & 101.24 & 100.67 & 102.37 & 100.44 & 100.50 & 97.15 \\
\hline IRAK1 & 100.81 & 100.16 & 102.79 & 101.36 & 98.92 & 97.90 \\
\hline IRAK4 & 88.83 & 88.31 & 82.71 & 78.47 & 88.61 & 84.94 \\
\hline IRR/INSRR & 123.57 & 121.01 & 108.49 & 99.85 & 121.06 & 113.76 \\
\hline ITK & 99.45 & 95.85 & 105.57 & 103.48 & 102.11 & 100.51 \\
\hline JAK1 & 96.67 & 96.26 & 102.44 & 99.53 & 101.38 & 99.07 \\
\hline JAK2 & 95.80 & 92.56 & 93.48 & 92.09 & 88.59 & 88.17 \\
\hline JAK3 & 102.64 & 101.59 & 106.09 & 102.92 & 100.59 & 99.31 \\
\hline JNK1 & 86.44 & 83.52 & 90.64 & 88.65 & 86.86 & 85.74 \\
\hline JNK2 & 91.34 & 90.94 & 93.42 & 91.99 & 94.72 & 94.14 \\
\hline JNK3 & 98.83 & 95.11 & 96.38 & 93.66 & 104.05 & 100.92 \\
\hline KDR/VEGFR2 & 97.27 & 95.43 & 95.86 & 92.99 & 88.36 & 85.59 \\
\hline KHS/MAP4K5 & 42.81 & 39.41 & 39.69 & 39.28 & 41.74 & 40.60 \\
\hline KSR1 & 93.57 & 92.67 & 94.48 & 93.83 & 96.28 & 95.91 \\
\hline KSR2 & 97.23 & 96.38 & 96.92 & 93.25 & 98.52 & 97.71 \\
\hline LATS1 & 101.28 & 99.48 & 97.36 & 97.31 & 102.26 & 100.53 \\
\hline LATS2 & 110.37 & 109.35 & 105.71 & 104.67 & 101.91 & 100.36 \\
\hline LCK & 96.99 & 96.67 & 93.16 & 92.53 & 100.83 & 98.93 \\
\hline LCK2/ICK & 89.44 & 86.13 & 107.87 & 104.57 & 97.53 & 95.60 \\
\hline LIMK1 & 77.94 & 76.31 & 65.09 & 64.18 & 74.16 & 72.63 \\
\hline LIMK2 & 105.28 & 102.22 & 102.34 & 99.63 & 97.82 & 95.75 \\
\hline LKB1 & 92.49 & 92.06 & 80.69 & 79.50 & 96.08 & 90.86 \\
\hline LOK/STK10 & 61.59 & 61.54 & 51.83 & 51.51 & 68.66 & 68.53 \\
\hline LRRK2 & 74.06 & 73.65 & 60.10 & 59.73 & 78.27 & 77.27 \\
\hline LYN & 84.03 & 82.25 & 79.87 & 79.23 & 91.04 & 88.67 \\
\hline LYN B & 95.08 & 91.23 & 99.25 & 96.56 & 98.25 & 97.80 \\
\hline MAK & 111.75 & 110.09 & 99.16 & 98.28 & 118.59 & 100.81 \\
\hline МАРКАРК2 & 99.92 & 98.48 & 103.16 & 101.22 & 95.40 & 93.08 \\
\hline МАРКАРКЗ & 99.55 & 97.50 & 99.51 & 99.20 & 97.25 & 96.39 \\
\hline MAPKAPK5/PRAK & 96.08 & 95.81 & 93.24 & 90.60 & 94.65 & 94.46 \\
\hline MARK1 & 92.51 & 91.67 & 88.02 & 87.63 & 94.12 & 89.90 \\
\hline MARK2/PAR-1Ba & 93.74 & 92.98 & 92.76 & 91.66 & 104.07 & 103.03 \\
\hline MARK3 & 93.00 & 91.46 & 83.46 & 82.91 & 94.97 & 93.66 \\
\hline MARK4 & 83.45 & 83.34 & 83.71 & 82.62 & 96.82 & 95.76 \\
\hline MAST3 & 97.45 & 97.29 & 94.66 & 93.52 & 95.55 & 95.17 \\
\hline MASTL & 94.94 & 93.05 & 99.27 & 98.59 & 102.39 & 100.30 \\
\hline MEK1 & 103.50 & 102.97 & 97.45 & 97.44 & 95.10 & 93.40 \\
\hline
\end{tabular}




\begin{tabular}{|c|c|c|c|c|c|c|}
\hline MEK2 & 91.30 & 89.42 & 87.11 & 85.87 & 93.98 & 91.58 \\
\hline MEK3 & 104.87 & 103.04 & 102.24 & 100.94 & 101.41 & 101.33 \\
\hline MEK5 & 96.31 & 93.44 & 86.86 & 85.95 & 93.15 & 92.97 \\
\hline MEKK1 & 89.43 & 87.93 & 94.26 & 92.92 & 97.67 & 97.31 \\
\hline MEKK2 & 82.92 & 81.13 & 95.51 & 95.02 & 100.62 & 98.82 \\
\hline MEKK3 & 99.81 & 92.94 & 97.97 & 93.57 & 108.60 & 100.00 \\
\hline MEKK6 & 95.17 & 89.86 & 98.28 & 96.75 & 95.93 & 95.16 \\
\hline MELK & 99.63 & 97.92 & 99.24 & 98.82 & 99.44 & 99.30 \\
\hline MINK/MINK1 & 37.11 & 36.56 & 24.06 & 22.65 & 30.16 & 29.65 \\
\hline MKK4 & 94.33 & 94.30 & 101.22 & 98.66 & 94.64 & 93.94 \\
\hline MKK6 & 68.55 & 64.71 & 84.36 & 80.71 & 79.73 & 79.33 \\
\hline MKK7 & 100.41 & 98.52 & 93.05 & 92.71 & 98.71 & 97.52 \\
\hline MLCK/MYLK & 106.35 & 106.15 & 106.34 & 106.15 & 103.44 & 101.49 \\
\hline MLCK2/MYLK2 & 82.26 & 81.15 & 66.82 & 66.59 & 84.82 & 82.74 \\
\hline MLK1/MAP3K9 & 103.62 & 102.91 & 111.75 & 110.44 & 99.78 & 98.95 \\
\hline MLK2/MAP3K10 & 115.37 & 106.44 & 86.36 & 86.29 & 98.53 & 96.07 \\
\hline MLK3/MAP3K11 & 106.15 & 101.95 & 99.36 & 99.32 & 96.73 & 96.66 \\
\hline MLK4 & 91.19 & 89.41 & 95.27 & 95.21 & 96.15 & 93.38 \\
\hline MNK1 & 60.95 & 59.43 & 51.11 & 50.34 & 71.38 & 70.81 \\
\hline MNK2 & 68.96 & 63.87 & 48.23 & 46.54 & 70.76 & 68.28 \\
\hline MRCKa/CDC42BPA & 104.45 & 102.98 & 102.59 & 102.07 & 105.33 & 104.72 \\
\hline MRCKb/CDC42BPB & 97.02 & 95.88 & 94.59 & 94.42 & 99.74 & 99.47 \\
\hline MSK1/RPS6KA5 & 99.70 & 96.47 & 87.13 & 82.15 & 90.51 & 89.44 \\
\hline MSK2/RPS6KA4 & 96.00 & 95.45 & 97.14 & 96.56 & 95.42 & 92.01 \\
\hline MSSK1/STK23 & 98.18 & 91.96 & 99.31 & 98.71 & 99.84 & 99.23 \\
\hline MST1/STK4 & 100.28 & 99.22 & 101.34 & 100.26 & 103.09 & 101.65 \\
\hline MST2/STK3 & 98.35 & 93.52 & 95.63 & 93.91 & 101.75 & 100.46 \\
\hline MST3/STK24 & 113.21 & 107.63 & 90.20 & 87.91 & 96.29 & 94.57 \\
\hline MST4 & 90.69 & 90.53 & 86.90 & 85.32 & 94.97 & 90.97 \\
\hline MUSK & 90.30 & 88.04 & 92.79 & 91.48 & 92.16 & 90.22 \\
\hline MYLK3 & 102.98 & 101.18 & 99.83 & 98.90 & 100.08 & 99.34 \\
\hline MYLK4 & 95.87 & 95.46 & 97.68 & 95.79 & 103.63 & 102.67 \\
\hline MYO3A & 99.37 & 95.48 & 84.03 & 81.86 & 94.74 & 94.68 \\
\hline MYO3b & 84.96 & 84.87 & 81.60 & 81.31 & 86.55 & 85.13 \\
\hline NEK1 & 100.04 & 93.20 & 87.56 & 84.54 & 88.09 & 84.46 \\
\hline NEK11 & 92.05 & 89.23 & 90.59 & 85.69 & 91.90 & 87.79 \\
\hline NEK2 & 93.02 & 91.46 & 92.28 & 90.39 & 98.45 & 96.23 \\
\hline NEK3 & 96.09 & 95.23 & 88.26 & 86.84 & 96.16 & 94.54 \\
\hline NEK4 & 100.85 & 99.62 & 98.49 & 97.67 & 98.05 & 97.25 \\
\hline
\end{tabular}




\begin{tabular}{|c|c|c|c|c|c|c|}
\hline NEK5 & 105.78 & 96.39 & 98.71 & 97.51 & 93.22 & 93.10 \\
\hline NEK6 & 100.84 & 100.11 & 99.26 & 98.81 & 103.64 & 103.36 \\
\hline NEK7 & 96.75 & 93.87 & 97.56 & 96.68 & 97.42 & 97.32 \\
\hline NEK8 & 101.75 & 100.17 & 98.40 & 98.06 & 109.21 & 106.54 \\
\hline NEK9 & 87.78 & 82.66 & 91.72 & 91.25 & 93.51 & 91.59 \\
\hline NIM1 & 94.49 & 93.25 & 96.64 & 95.18 & 98.15 & 97.81 \\
\hline NLK & 44.71 & 44.67 & 21.99 & 20.81 & 50.44 & 47.74 \\
\hline OSR1/OXSR1 & 96.08 & 95.05 & 93.41 & 93.34 & 94.97 & 94.75 \\
\hline P38a/MAPK14 & 99.75 & 99.05 & 92.73 & 91.67 & 97.01 & 96.02 \\
\hline P38b/MAPK11 & 101.90 & 99.99 & 92.29 & 92.20 & 95.54 & 95.51 \\
\hline P38d/MAPK13 & 103.50 & 101.10 & 100.64 & 98.26 & 97.65 & 97.52 \\
\hline P38g & 94.38 & 90.04 & 109.56 & 107.36 & 107.64 & 105.83 \\
\hline p70S6K/RPS6KB1 & 97.82 & 94.87 & 97.80 & 96.69 & 100.22 & 99.76 \\
\hline p70S6Kb/RPS6KB2 & 102.82 & 98.03 & 97.27 & 97.21 & 101.90 & 99.09 \\
\hline PAK1 & 103.65 & 103.63 & 104.85 & 102.29 & 103.23 & 100.98 \\
\hline PAK2 & 106.75 & 104.17 & 100.73 & 100.35 & 104.48 & 103.95 \\
\hline PAK3 & 90.17 & 89.47 & 89.45 & 87.14 & 124.51 & 124.21 \\
\hline PAK4 & 103.71 & 103.30 & 98.47 & 98.21 & 99.91 & 98.82 \\
\hline PAK5 & 96.74 & 96.36 & 94.56 & 92.74 & 97.29 & 94.99 \\
\hline PAK6 & 98.78 & 98.70 & 102.00 & 97.22 & 96.32 & 95.67 \\
\hline PASK & 90.71 & 89.01 & 93.95 & 93.23 & 99.15 & 98.21 \\
\hline PBK/TOPK & 95.05 & 94.31 & 91.79 & 89.81 & 102.69 & 102.56 \\
\hline PDGFRa & 80.05 & 79.03 & 82.87 & 81.22 & 81.89 & 81.08 \\
\hline PDGFRb & 90.14 & 88.87 & 77.30 & 76.01 & 92.12 & 89.56 \\
\hline PDK1/PDPK1 & 96.26 & 93.89 & 94.32 & 91.49 & 95.91 & 94.36 \\
\hline PEAK1 & 112.02 & 110.20 & 99.07 & 97.59 & 96.57 & 95.56 \\
\hline PHKg1 & 96.68 & 92.81 & 85.63 & 82.79 & 96.97 & 95.24 \\
\hline PHKg2 & 95.85 & 95.06 & 94.94 & 93.32 & 96.68 & 96.43 \\
\hline PIM1 & 105.63 & 103.63 & 100.02 & 97.92 & 95.25 & 92.25 \\
\hline PIM2 & 98.05 & 96.36 & 99.17 & 95.51 & 99.70 & 98.82 \\
\hline PIM3 & 107.18 & 106.04 & 100.03 & 99.10 & 107.12 & 106.19 \\
\hline PKA & 97.99 & 97.30 & 97.90 & 96.94 & 96.87 & 95.62 \\
\hline PKAcb & 99.42 & 98.30 & 96.17 & 95.19 & 97.92 & 96.20 \\
\hline PKAcg & 91.97 & 88.31 & 94.08 & 93.99 & 100.89 & 98.97 \\
\hline PKCa & 88.30 & 85.60 & 89.76 & 89.62 & 90.26 & 89.76 \\
\hline PKCb1 & 87.68 & 86.84 & 85.45 & 82.63 & 85.35 & 83.58 \\
\hline PKCb2 & 104.64 & 103.20 & 106.80 & 106.51 & 99.11 & 97.56 \\
\hline PKCd & 93.40 & 92.22 & 98.99 & 97.72 & 101.05 & 100.12 \\
\hline PKCepsilon & 97.83 & 97.53 & 99.77 & 96.64 & 106.14 & 105.76 \\
\hline
\end{tabular}




\begin{tabular}{|c|c|c|c|c|c|c|}
\hline PKCeta & 96.80 & 96.60 & 94.91 & 93.90 & 98.29 & 98.10 \\
\hline PKCg & 98.34 & 97.90 & 97.01 & 96.27 & 97.16 & 96.41 \\
\hline PKCiota & 100.39 & 95.18 & 107.22 & 100.36 & 99.14 & 98.91 \\
\hline PKCmu/PRKD1 & 93.33 & 90.68 & 90.08 & 88.26 & 97.61 & 95.09 \\
\hline PKCnu/PRKD3 & 144.24 & 142.24 & 83.26 & 82.18 & 89.59 & 89.04 \\
\hline PKCtheta & 102.63 & 95.99 & 85.06 & 81.31 & 87.00 & 84.96 \\
\hline PKCzeta & 92.41 & 91.33 & 91.17 & 90.40 & 92.51 & 91.43 \\
\hline PKD2/PRKD2 & 105.17 & 102.86 & 96.07 & 95.72 & 99.15 & 98.89 \\
\hline PKG1a & 103.94 & 103.73 & 97.90 & 97.81 & 101.80 & 101.45 \\
\hline PKG1b & 99.52 & 98.95 & 101.57 & 98.83 & 98.81 & 96.31 \\
\hline PKG2/PRKG2 & 95.65 & 91.81 & 98.88 & 97.48 & 98.49 & 97.08 \\
\hline PKMYT1 & 124.81 & 121.94 & 101.28 & 100.09 & 94.46 & 92.38 \\
\hline PKN1/PRK1 & 94.64 & 94.01 & 98.54 & 96.76 & 98.68 & 96.39 \\
\hline PKN2/PRK2 & 102.96 & 100.36 & 94.53 & 91.84 & 95.93 & 95.77 \\
\hline PKN3/PRK3 & 100.61 & 98.77 & 95.02 & 93.55 & 98.38 & 96.44 \\
\hline PLK1 & 98.82 & 97.79 & 92.38 & 91.24 & 92.72 & 92.70 \\
\hline PLK2 & 93.59 & 92.87 & 98.76 & 97.24 & 102.70 & 102.38 \\
\hline PLK3 & 96.05 & 93.88 & 94.99 & 93.70 & 97.67 & 94.47 \\
\hline PLK4/SAK & 94.00 & 93.89 & 94.12 & 93.32 & 98.82 & 98.79 \\
\hline PRKX & 96.97 & 96.87 & 99.94 & 99.24 & 91.29 & 90.55 \\
\hline PYK2 & 83.76 & 81.78 & 85.92 & 85.65 & 88.59 & 85.73 \\
\hline RAF1 & 85.03 & 84.08 & 78.66 & 78.57 & 85.46 & 83.85 \\
\hline RET & 92.55 & 89.18 & 94.44 & 94.34 & 96.01 & 93.64 \\
\hline RIPK2 & 80.92 & 79.92 & 71.12 & 66.31 & 89.66 & 89.10 \\
\hline RIPK3 & 112.17 & 111.71 & 106.23 & 101.40 & 103.87 & 102.83 \\
\hline RIPK4 & 79.68 & 77.02 & 75.50 & 70.28 & 86.09 & 82.04 \\
\hline RIPK5 & 84.75 & 83.86 & 83.14 & 82.21 & 86.85 & 86.47 \\
\hline ROCK1 & 96.97 & 96.20 & 94.95 & 91.98 & 94.71 & 92.61 \\
\hline ROCK2 & 121.91 & 119.86 & 115.65 & 115.13 & 100.51 & 93.98 \\
\hline RON/MST1R & 113.67 & 108.77 & 98.74 & 97.00 & 105.33 & 104.29 \\
\hline ROS/ROS1 & 95.56 & 95.52 & 87.73 & 86.47 & 97.39 & 95.54 \\
\hline RSK1 & 97.01 & 95.84 & 98.93 & 97.99 & 101.97 & 101.00 \\
\hline RSK2 & 97.43 & 97.24 & 100.90 & 99.92 & 102.57 & 101.06 \\
\hline RSK3 & 101.40 & 99.21 & 92.16 & 87.93 & 101.35 & 99.74 \\
\hline RSK4 & 97.30 & 94.31 & 95.95 & 95.64 & 95.46 & 94.61 \\
\hline SBK1 & 96.31 & 96.20 & 94.60 & 93.74 & 103.20 & 101.08 \\
\hline SGK1 & 91.09 & 90.95 & 94.93 & 93.48 & 98.75 & 95.22 \\
\hline SGK2 & 95.78 & 94.93 & 102.03 & 100.43 & 102.54 & 100.91 \\
\hline SGK3/SGKL & 86.00 & 83.45 & 102.87 & 99.35 & 94.81 & 93.33 \\
\hline
\end{tabular}




\begin{tabular}{|c|c|c|c|c|c|c|}
\hline SIK1 & 88.51 & 87.47 & 82.87 & 82.71 & 93.76 & 91.60 \\
\hline SIK2 & 36.68 & 35.99 & 31.97 & 30.68 & 43.90 & 43.50 \\
\hline SIK3 & 72.61 & 71.66 & 59.47 & 58.19 & 88.98 & 82.93 \\
\hline SLK/STK2 & 105.63 & 104.60 & 100.24 & 96.16 & 100.70 & 100.64 \\
\hline SNARK/NUAK2 & 93.54 & 92.65 & 93.71 & 91.64 & 94.38 & 93.96 \\
\hline SNRK & 113.18 & 107.97 & 105.10 & 104.94 & 100.30 & 97.75 \\
\hline SRMS & 98.82 & 97.08 & 96.58 & 95.09 & 96.08 & 95.91 \\
\hline SRPK1 & 104.82 & 102.86 & 111.76 & 105.40 & 89.32 & 81.70 \\
\hline SRPK2 & 97.17 & 95.75 & 97.75 & 94.13 & 121.46 & 115.95 \\
\hline SSTK/TSSK6 & 110.28 & 107.83 & 110.04 & 107.86 & 98.18 & 98.04 \\
\hline STK16 & 102.11 & 99.20 & 106.91 & 103.69 & 100.72 & 99.45 \\
\hline STK21/CIT & 102.54 & 99.05 & 88.76 & 84.42 & 89.07 & 84.97 \\
\hline STK22D/TSSK1 & 101.05 & 99.56 & 97.48 & 97.01 & 100.64 & 99.16 \\
\hline STK25/YSK1 & 92.82 & 91.63 & 95.70 & 94.98 & 96.14 & 94.06 \\
\hline STK32B/YANK2 & 86.51 & 82.92 & 87.61 & 87.20 & 98.22 & 94.20 \\
\hline STK32C/YANK3 & 92.80 & 90.70 & 86.77 & 85.01 & 93.31 & 91.96 \\
\hline STK33 & 97.29 & 96.42 & 91.03 & 88.77 & 97.37 & 96.94 \\
\hline STK38/NDR1 & 91.48 & 88.77 & 91.87 & 90.38 & 96.68 & 93.15 \\
\hline STK38L/NDR2 & 95.32 & 93.58 & 99.37 & 98.69 & 103.76 & 102.97 \\
\hline STK39/STLK3 & 97.61 & 94.07 & 89.10 & 88.76 & 95.70 & 93.62 \\
\hline SYK & 104.83 & 99.79 & 101.32 & 100.12 & 101.79 & 101.24 \\
\hline TAK1 & 98.69 & 97.41 & 98.52 & 97.44 & 99.08 & 98.46 \\
\hline TAOK1 & 38.45 & 36.37 & 60.28 & 60.19 & 62.19 & 60.59 \\
\hline TAOK2/TAO1 & 82.27 & 81.16 & 88.57 & 87.58 & 100.90 & 99.37 \\
\hline TAOK3/JIK & 68.90 & 65.47 & 69.70 & 69.26 & 84.52 & 81.51 \\
\hline TBK1 & 87.00 & 83.46 & 93.61 & 92.56 & 87.18 & 85.62 \\
\hline TEC & 127.66 & 124.33 & 107.92 & 107.42 & 106.34 & 106.19 \\
\hline TESK1 & 108.31 & 108.28 & 113.76 & 108.88 & 105.36 & 105.23 \\
\hline TESK2 & 93.29 & 93.23 & 93.94 & 93.89 & 92.15 & 92.03 \\
\hline TGFBR2 & 111.40 & 110.01 & 102.91 & 97.44 & 105.82 & 105.33 \\
\hline TIE2/TEK & 93.07 & 91.97 & 93.84 & 92.85 & 93.36 & 92.15 \\
\hline TLK1 & 111.42 & 109.03 & 108.27 & 107.91 & 103.74 & 100.84 \\
\hline TLK2 & 95.15 & 94.99 & 96.63 & 95.61 & 93.27 & 89.84 \\
\hline TNIK & 5.27 & 4.57 & -0.77 & -2.62 & 4.85 & 3.92 \\
\hline TNK1 & 108.03 & 102.51 & 105.47 & 99.53 & 98.56 & 98.22 \\
\hline TRKA & 91.94 & 89.07 & 91.84 & 90.11 & 95.24 & 94.00 \\
\hline TRKB & 109.41 & 105.99 & 100.49 & 99.94 & 98.97 & 98.44 \\
\hline TRKC & 118.59 & 117.99 & 102.66 & 101.44 & 99.02 & 97.50 \\
\hline TSSK2 & 107.44 & 102.75 & 100.20 & 98.77 & 100.08 & 99.71 \\
\hline
\end{tabular}




\begin{tabular}{|c|c|c|c|c|c|c|}
\hline TSSK3/STK22C & 95.08 & 94.89 & 96.57 & 96.02 & 96.56 & 95.64 \\
\hline TTBK1 & 96.89 & 93.79 & 97.29 & 96.78 & 99.41 & 98.52 \\
\hline TTBK2 & 97.99 & 94.77 & 97.12 & 94.62 & 97.80 & 96.48 \\
\hline TXK & 103.85 & 100.24 & 101.32 & 100.80 & 98.23 & 95.85 \\
\hline TYK1/LTK & 100.49 & 99.87 & 99.76 & 99.01 & 99.99 & 99.29 \\
\hline TYK2 & 103.25 & 100.78 & 92.64 & 91.37 & 102.60 & 99.47 \\
\hline TYRO3/SKY & 97.39 & 95.17 & 84.13 & 83.48 & 94.52 & 93.66 \\
\hline ULK1 & 86.28 & 82.29 & 84.69 & 83.53 & 89.77 & 89.24 \\
\hline ULK2 & 81.06 & 80.11 & 73.70 & 73.24 & 85.24 & 84.46 \\
\hline ULK3 & 94.55 & 93.28 & 91.50 & 91.23 & 98.85 & 97.24 \\
\hline VRK1 & 108.53 & 108.29 & 104.27 & 100.69 & 101.56 & 98.74 \\
\hline VRK2 & 88.02 & 85.61 & 89.06 & 87.47 & 92.46 & 89.63 \\
\hline WEE1 & 128.65 & 120.95 & 104.70 & 104.15 & 104.62 & 99.73 \\
\hline WNK1 & 101.95 & 100.13 & 107.57 & 107.33 & 104.49 & 102.29 \\
\hline WNK2 & 98.86 & 98.34 & 95.45 & 95.09 & 96.93 & 93.64 \\
\hline WNK3 & 92.11 & 90.95 & 89.56 & 88.15 & 93.78 & 92.59 \\
\hline YES/YES1 & 100.50 & 98.27 & 104.81 & 104.56 & 109.01 & 108.87 \\
\hline YSK4/MAP3K19 & 105.08 & 101.54 & 98.16 & 97.39 & 97.00 & 96.61 \\
\hline ZAK/MLTK & 38.53 & 36.39 & 31.26 & 30.86 & 46.64 & 46.59 \\
\hline ZAP70 & 99.62 & 98.79 & 96.23 & 94.79 & 98.70 & 98.47 \\
\hline ZIPK/DAPK3 & 108.04 & 105.01 & 103.90 & 103.31 & 103.72 & 102.34 \\
\hline
\end{tabular}

